\title{
Nonlinear Games: examples and counterexamples
}

\author{
"It would be more impressive if it flowed the other way." \\ Oscar Wilde at Niagara Falls
}

\author{
John Doyle ${ }^{1}$ \\ doyle@hot.caltech.edu
}

\author{
James A. Primbs ${ }^{1}$ \\ jprimbs@hot.caltech.edu
}

\author{
Benjamin Shapiro ${ }^{1}$ \\ bshapiro@indra.caltech.edu
}

\author{
Vesna Nevistić ${ }^{2}$ \\ vesnaQaut.ee.ethz.ch
}

\begin{abstract}
In this session, popular nonlinear control methodologies are compared using benchmark examples generated with a "converse Hamilton-Jacobi-Bellman" method (CoHJB). Starting with the cost and optimal value function V, CoHJB solves HJB PDEs "backwards" algebraically to produce nonlinear dynamics and optimal controllers and disturbances. Although useless for design, it is great for generating benchmark examples. It is easy to use, computational tractable, and can generate essentially all possible nonlinear optimal control problems. The optimal control and disturbance are then known and can be used to study actual design methods, which must start with the cost and dynamics without knowledge of $\mathrm{V}$.
\end{abstract}

This paper gives a brief introduction to the CoHJB method and some of the ground rules for comparing various methods. Some very simple examples are given to illustrate the main ideas. Both Jacobian linearization and feedback linearization combined with linear optimal control are used as "strawmen" design methods. Other more sophisticated techniques are presented in the remaining papers in this session. More details, including cross-comparisons not available in the individual papers, can be found from the web page: http://hot.caltech.edu/ doyle.

\section{Background}

The motivation for this paper and the session it prefaces is that we want to deepen our understanding of nonlinear control design, and particularly the relationship between some of the more popular nonlinear control design methodologies. We want to do this through examples that are generated independently from the methodologies themselves. Since as usual we learn

\footnotetext{
${ }^{1}$ Control and Dynamical Systems, California Institute of Technology, Pasadena, CA 91125. This work was supported by AFOSR and NSF.

${ }^{2}$ Automatic Control Laboratory, Swiss Federal Institute of Technology (ETH), CH-8092 Zürich, Switzerland.
}

much more from failure than success, we want a systematic technique to generate examples which are hard for a given design method. Essentially, we want a counterexample generating methodology. The typical published "application" in most papers is simply a walkthrough of the theory with numbers in place of symbols. If the theory works for symbols then it should work for numbers. This may have some pedagogical value, but it rarely teaches us anything deep about the method. Even more practical applications often have limited value unless they point out difficulties with applying a method.

A carefully constructed counterexample can reveal a great deal. Counterexamples are most often used to show a conjecture or folk theorem to be false. Ideally, a counterexample should be both trivial and obvious, often making it look contrived. If a counterexample becomes too involved, then it becomes difficult to determine exactly where the problem is and to what exactly it is a counterexample. Unfortunately, for our purposes the examples must be complicated enough that they challenge the design methodologies. More precisely, they must appear complicated from the point of view adopted by a particular methodology. Thus we want a way to allow for a simplicity that can be hidden from the designer.

We also want a problem description which would be easy if it so happened that the example were linear. We recognize that this greatly limits inclusion of practical issues like plant uncertainty and controller structure constraints, but it has the advantage of focusing on nonlinearity alone as the source of difficulty.

This paper does not propose any particular nonlinear design method, but we will use Jacobian linearization and feedback linearization combined with linear optimal control as test cases to illustrate the ways in which we can analyze a given design methodology. These will be used to provide baseline designs against which the other methods can be compared. The other papers in this session then each advocate a particular design or analysis technique and we will use the methods of this 
paper to generate examples and counterexamples for these methods.

\section{A Nonlinear Optimal Control Problem}

Consider the nonlinear system, with $f(0)=0$

$$
\dot{x}(t)=f(x(t))+g_{1}(x(t)) w(t)+g_{2}(x(t)) u(t)
$$

and performance objective

$$
\begin{gathered}
\inf _{u} \sup _{w} J(u, w) \\
J(u, w)=\int_{o}^{\infty}\left(q(x(t))+u^{T}(t) u(t)-w^{T}(t) w(t)\right) d t
\end{gathered}
$$

We can use this framework to consider various problems:

1. stability analysis $\left(g_{1}=0, g_{2}=0\right)$

2. analysis of disturbance rejection $\left(g_{2}=0\right)$

3. stabilization with minimum $\|u\|_{2}\left(g_{1}=0, q=0\right)$

4. stabilization with quadratic cost $\left(g_{1}=0\right)$

5. "Nonlinear $H_{\infty}$ "

In the following, we consider the case where the state $x$ is available for the control $u$. The above problem can be solved as a differential game [1]. A sufficient condition for the above optimal control is that there exists a $\mathcal{C}^{1}$ nonnegative function $V: \mathbf{R}^{n} \rightarrow \mathbf{R}^{+}$such that the following Hamilton-Jacobi-Bellman partial differential equation (HJE) is satisfied, where $V_{x}=\frac{\partial V(x)}{\partial x}$

$$
\begin{gathered}
0=\min _{u \in \mathbb{R}^{m_{1}}} \max _{w \in \mathbb{R}^{m_{2}}}\left\{V_{x}\left(f(x)+g_{1}(x) w+g_{2}(x) u\right)\right. \\
\left.+q(x)+u^{T} u-w^{T} w\right\} \\
=\min \max \left\{\left\|u+\frac{1}{2} g_{2}^{T} V_{x}^{T}\right\|^{2}-\left\|w-\frac{1}{2} g_{1}^{T} V_{x}^{T}\right\|^{2}\right. \\
\left.+V_{x} f+\frac{1}{4} V_{x}\left(g_{1} g_{1}^{T}-g_{2} g_{2}^{T}\right) V_{x}^{T}+q\right\} .
\end{gathered}
$$

The optimal is achieved by

$$
u^{*}(x)=-\frac{1}{2} g_{2}^{T}(x) V_{x}^{T}, \quad w^{*}(x)=\frac{1}{2} g_{1}^{T}(x) V_{x}^{T},
$$

and the HJE becomes

$$
V_{x} f+\frac{1}{4} V_{x}\left(g_{1} g_{1}^{T}-g_{2} g_{2}^{T}\right) V_{x}^{T}+q=0,
$$

which gives the optimal solution.
As noted above, we can solve various problems, such as stabilization, or nonlinear versions of $\mathrm{LQR}$ and $\mathcal{H}_{\infty^{-}}$ control, in the above framework. We will require that each method in this session address one of the problems outlined above, and preferably the most general. While this is somewhat artificial, it creates a level playing field and an optimal cost for comparison. The potential difficulty with this approach is that this optimal can only be computed in special cases, such as linear dynamics and quadratic cost, or very low dimensional systems. Basically, to compute the optimal controller for (2) we must solve the HJE (4) with $V(0)=0$, and the controller is then constructed as (3). The HJEs are partial differential equations (PDEs), and no efficient algorithm is available to solve the PDE when it is nonlinear and the problem dimension is high. This is of course why reducing our problem to "solving" HJEs cannot be viewed as a general, practical method. Nevertheless, for our whole session to be successful we must be able to compute the optimal. This difficulty is overcome by using a converse HJB method.

\section{Converse Optimal Control}

The term inverse optimal control problem is usually concerned with finding for what cost function some given controller for a given system is optimal. To avoid confusion with this terminology, we will use the term converse problem to the optimal control problem (1)(2) defined as follows:

Given a performance defined as (2) and a storage function $V: \mathbf{R}^{n} \rightarrow \mathbf{R}^{+}$, find a class of (nonlinear) systems such that the optimal control problem (2) has this as its solution.

The converse problem is also characterized by the HJE, but its role is reversed. The converse problem requires only solving the HJE (4) as an algebraic equation in the unknowns $f, g_{1}, g_{2}$ with $V$ given, rather than a PDE in unknown $V$ with $f, g_{1}, g_{2}$ given. In addition to providing insights into the original optimal control problem, the converse problem helps to construct an array of examples which have known optimal solutions. Note that essentially any nonlinear optimal control problem of the type described above can be generated with this method. Unfortunately, this is not useful for actual design when $f, g$, and $q$ are given.

In the following, we will consider the converse problem by examining some examples. To make the exposition concise but without loss of generality, we only consider the case of no disturbances $\left(g_{1}=0\right)$, so the only uncertainty is initial conditions. For simplicity, we will 
mostly use quadratic costs and quadratic storage functions but the method is in no way limited to these. We will also use nonlinear functions that make the equations easy to read, such as $e^{x}, \cos (x)$, polynomials, etc. which can make the examples quite extreme. Again, the method is not limited to such simple nonlinearities.

As we will see, even with these restrictions we can have interesting nonlinear behavior. Although the examples in this paper do not have explicit physical motivation, it is not hard to generate examples which have similar characteristics and can be given physical motivation. Unfortunately, this greatly increases the space required beyond what is available. Even though the examples studied here are "just" mathematical, it would be very wishful thinking to hope that the difficulties they present for nonlinear design would not arise in more physically motivated examples.

\subsection{Linear Systems}

$$
\dot{x}=A x+B u
$$

If $q(x)=x^{T} Q x$ and $V(x)=x^{T} x$, the HJE reduces to the Riccati equation

$$
A+A^{T}-B B^{T}+Q=0
$$

or

$$
A=\frac{1}{2}\left(-Q+B B^{T}\right)+S
$$

where $S$ is any skew matrix, $\left(S+S^{T}=0\right)$.

\subsection{1-D Systems}

$$
\dot{x}=f(x)+g(x) u
$$

If $q(x)=x^{2}$ and $V(x)=x^{2}$, the HJE is

$$
V_{x} f(x)-\frac{1}{4}\left(V_{x}\right)^{2} g^{2}(x)+x^{2}=0
$$

Then $f(x)=-\frac{1}{2} x\left(1-g^{2}(x)\right), u^{*}(x)=-g(x) x$, and the closed loop system is $\dot{x}=-\frac{1}{2} x\left(1+g^{2}(x)\right) x$ where $g(x)$ can be any function.

Next, we consider a slightly different converse problem. Let $g(x)>0$ for all $x \in \mathbf{R}$, and suppose we require $u^{*}(x)=-x$ to be the optimal solution. We are asking for what nonlinear systems is this linear controller optimal. Since the optimal state feedback is $u^{*}(x)=-\frac{1}{2} g(x) V_{x}$, then

$$
\frac{\partial V(x)}{\partial x}=\frac{2 x}{g(x)} .
$$

If we take $q(x)=q x^{2}$ with $q>0$, then the HJE is

$$
\frac{2 x}{g(x)} f(x)=\frac{x^{2}}{g^{2}(x)} g^{2}(x)-q x^{2}
$$

which can be solved for $f(x)=(1-q) g(x) x$, and the closed loop system is $\dot{x}=-q g(x) x$.

\subsection{2-D Oscillators}

Consider the following system:

$$
\left\{\begin{array}{l}
\dot{x}_{1}=x_{2} \\
\dot{x}_{2}=f(x)+g(x) u
\end{array}\right.
$$

and suppose that $q(x)=x_{2}^{2}$ and $V(x)=x_{1}^{2}+x_{2}^{2}$.

The HJE is (with $V_{i}=\frac{\partial V(x)}{\partial x_{i}}$ )

$$
V_{1} x_{2}+V_{2} f(x)-\frac{1}{4}\left(V_{2}\right)^{2} g^{2}(x)+q(x)=0
$$

or

$$
2 x_{1} x_{2}+2 x_{2} f(x)=\frac{1}{4}\left(2 x_{2}\right)^{2} g^{2}(x)-x_{2}^{2} .
$$

Therefore

$$
f(x)=-x_{1}-\frac{1}{2} x_{2}\left(1-g^{2}(x)\right),
$$

the dynamics are

$$
\left\{\begin{array}{l}
\dot{x}_{1}=x_{2} \\
\dot{x}_{2}=-x_{1}-\frac{1}{2} x_{2}\left(1-g^{2}(x)\right)+g(x) u
\end{array}\right.
$$

and the optimal control is $u^{*}(x)=-g x_{2}$. Note that we were able to constrain the form of the dynamics in 6. An interesting example is $g(x)=x_{1}$ which yields a Van der Pol oscillator with a stable but linearly uncontrollable equilibrium at the origin and an unstable limit cycle. Other interesting nonlinear oscillators can be constructed with other choices of $g$ and nonlinear terms can be added to the "frequency" and so on.

\subsection{General Nonlinear Systems}

Consider the following system

$$
\dot{x}=f(x)+g(x) u,
$$

with $x \in \mathbf{R}^{n}$. Suppose $q(x)=x^{T} Q x$ and $V(x)=x^{T} P x$ with $P>0$, so that

$$
\frac{\partial V(x)}{\partial x}=2 x^{T} P
$$

The HJE is

$$
2 x^{T} P f(x)-x^{T} P g(x) g^{T}(x) P x+x^{T} Q x=0 .
$$

If

$$
f(x)=\frac{1}{2}\left(g(x) g^{T}(x) P-P^{-1} Q\right) x+P^{-1} \gamma(x)
$$

with $\gamma: \mathbf{R}^{n} \rightarrow \mathbf{R}^{n}, \gamma(0)=0$ satisfying $x^{T} \gamma(x)=0$ for all $x \in \mathbf{R}^{n}$, then (8) is satisfied. In this case, the optimal control is $u^{*}(x)=-g^{T}(x) P x$. We can choose $g(x)$ and $\gamma(x)$ to make the dynamics highly nonlinear, yet the optimal $V$ is quadratic and the controller is known. 


\section{Design examples}

To further illustrate the converse approach, we will generate nonlinear examples to which we will apply linear optimal control combined with Jacobian and feedback linearization. We will Jacobian linearize the nonlinear system at $x=0$ and simply apply linear optimal control with the quadratic portion of the cost (neglecting higher order terms). As a more sophisticated alternative, we will then further use feedback linearization to force these optimal linear closed-loop dynamics to hold globally, and then solve for the controller required. We will only consider globally feedback linearizable systems.

\subsection{1-D example}

In one dimension, all examples are essentially trivial and don't require the converse methods, since the HJE is itself trivial to solve. For example, consider the system

$$
\dot{x}=e^{x} u
$$

The HJE is given by

$$
x^{2}-\frac{1}{4}\left(V_{x}\right)^{2} e^{2 x}=0
$$

so $V_{x}=2 x e^{-x}$ and the optimal control law is

$$
u^{*}=-x \text {. }
$$

Interestingly enough, we get this same control law by Jacobian Linearization. The storage function is not quadratic:

$$
V(x)=J_{J L}(x)=-2(1+x) e^{-x}+2 .
$$

The optimal closed loop dynamics are $\dot{x}=-e^{x} x$.

On the other hand, the controller designed by using feedback linearization is $u_{F L}=-x e^{-x}$ and results in the linear closed loop $\dot{x}=-x$. Even though this controller is locally optimal (i.e. optimal for the linearized system), it is immediately obvious from comparison with (10) that $u_{F L}$ is very different from $u^{*}$ for large $|x|$. The cost for this controller is given by:

$$
J_{F L}(x)=\frac{1}{4}\left(1-e^{-2 x}-2 x e^{-2 x}+2 x^{2}\right) .
$$

Notice that the optimal cost (11) grows as $-x e^{-x}$ for $x \rightarrow-\infty$ and asymptotically approaches 2 as $x \rightarrow \infty$. Meanwhile, the FL cost (12) grows as $-x e^{-2 x}$ for $x \rightarrow-\infty$ and as $x^{2}$ for $x \rightarrow \infty$. The FL based design is arbitrarily worse than optimal for $|x|$ sufficiently large. One often hears nonlinear control folktales that say feedback linearization is dubious because it cancels "beneficial" drift nonlinearities. This example has no drift term and focuses attention on the much more serious problem created by the inversion of $g$.
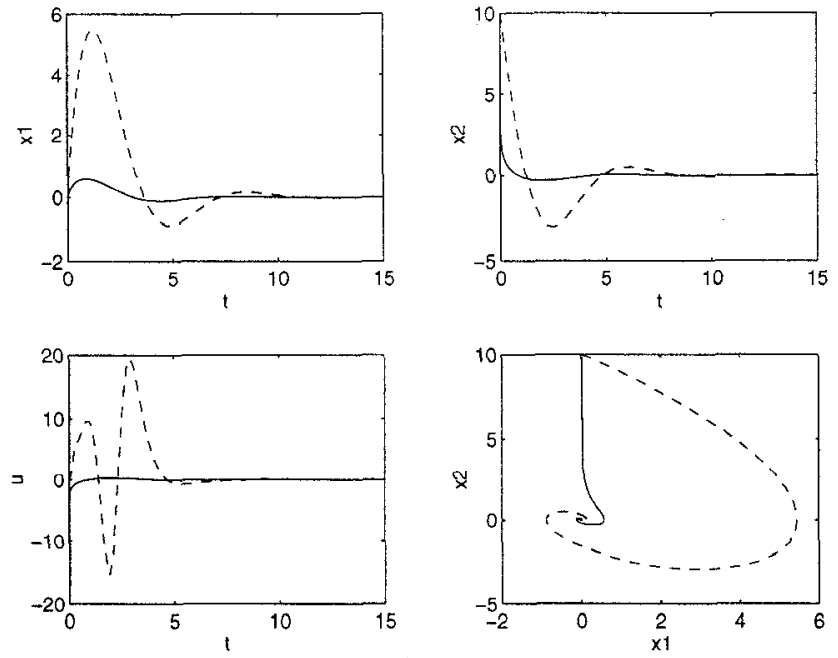

Figure 1: Comparison: Jacobian Linearization (solid) $J_{J L}=2$, Feedback Linearization (dashed) $J_{F L}=520$

\subsection{A 2-D nonlinear oscillator}

As a slightly less trivial example consider:

$$
\left\{\begin{array}{l}
\dot{x}_{1}=x_{2} \\
\dot{x}_{2}=-2 e^{x_{2}} x_{1}+e^{x_{2}} \cos \left(x_{1}\right) \sin \left(x_{1}\right)+e^{x_{2}} u
\end{array}\right.
$$

with performance objective:

$$
J=\int_{0}^{\infty} x_{2}^{2}+u^{2}
$$

The JL control is $u_{J L}=-x_{2}$ while the FL controller is $u_{F L}=-\left(e^{-x_{2}}\right)\left(-2 e^{x_{2}} x_{1}+e^{x_{2}} \cos \left(x_{1}\right) \sin \left(x_{1}\right)+x_{1}+x_{2}\right)$

Simulation results are shown for the initial condition $(0,10)$ in Figure 1. The JL design (cost=2) substantially outperforms the FL design (cost $=520$ ). As we will see, the JL controller was actually the same as the nonlinear optimal for this problem. Again, the explanation that the FL design does not perform well because it cancels "beneficial" drift nonlinearities does not apply. For example, the control

$$
u=-\left(e^{-x_{2}}\right)\left(-2 e^{x_{2}} x_{1}+e^{x_{2}} \cos \left(x_{1}\right) \sin \left(x_{1}\right)\right)-x_{1}-x_{2}
$$

cancels the same nonlinearities as $u_{F L}$, but simulation results indicate that this controller performs much closer to the optimal. The cost for the above initial conditions was within $1 \%$ of optimal.

\section{Optimality of Design Techniques}

You might have guessed that the examples treated in the previous section were biased toward Jacobian lin- 
earization. In fact, they were constructed so that Jacobian linearization would give the optimal control action. Using a converse HJB approach, it is possible to classify when different nonlinear design techniques will be optimal. In 1-D these characterizations often take a simple form.

\subsection{1-D examples}

For the scalar dynamics in (5) and $q(x)=x^{2}$ the following conclusions about Jacobian linearization and feedback linearization are easily derived from the HJE:

i) Jacobian linearization (JL) is optimal if and only if

$$
\frac{f^{\prime}(0)}{g(0)} x=\frac{f(x)}{g(x)}
$$

ii)Feedback linearization (FL) is optimal if and only if

$$
f^{2}(x)-x^{2}\left[f^{\prime}(0)\right]^{2}+x^{2}\left[g^{2}(x)-g^{2}(0)\right]=0 .
$$

From the above, it is easy to generate some simple examples where feedback linearization or Jacobian linearization is optimal. The table below lists a couple of these cases:

Table 1

\begin{tabular}{|l||l|l|}
\hline & $g(x)$ for FL & $g(x)$ for JL \\
\hline \hline$f(x)=0$ & constant & arbitrary \\
\hline$f(x)$ linear & constant & constant \\
\hline$f(x)=x e^{x}$ & $\sqrt{1-e^{2 x}+g^{2}(0)}$ & $e^{x}$ \\
& Only defined & Defined $\forall x$ \\
& $x<\frac{1}{2} \ln \left(1+g^{2}(0)\right)$ & \\
\hline
\end{tabular}

The first row shows that if $f(x)$ is zero, then $\mathrm{JL}$ is optimal for any $g(x)$ while an FL based control design is optimal only when $g(x)$ is constant. We used this fact for our 1-D example.

The third row is an example that shows that we can only have FL optimal on some range of $x$. This is true in a more general sense. For FL to be optimal, the following must hold,

$$
\begin{aligned}
& f(x)=x \sqrt{f^{\prime 2}(0)+g^{2}(0)-g^{2}(x)} \\
& g(x)=\sqrt{g^{2}(0)+f^{\prime 2}(0)-\frac{f^{2}(x)}{x^{2}}} .
\end{aligned}
$$

We see that if FL is to be optimal, $|g(x)|$ must be bounded for all $x$ (i.e. $|g(x)| \leq \sqrt{g^{2}(0)+f^{\prime 2}(0)}$ ) while $f(x)$ must be bounded by a linear function, $(|f(x)|<$ $\left.\sqrt{f^{\prime 2}(0)+g^{2}(0)}|x|\right)$ for all $x$. If this is not the case then the square roots can be undefined. This is not the case for JL.
If we actually wish to generate systems such that a specific design technique will be optimal, then by approaching the problem in a slightly different way, we can determine what $f$ and $g$ must be in terms of $q$ and the optimal value function $V$.

\subsection{The 2D oscillator revisited}

For the $2 \mathrm{D}$ oscillator, we are making a priori constraints on the nonlinear dynamics by insisting that $\dot{x}_{1}=x_{2}$. Thus for the converse problem to make sense (i.e. be solvable), $V$ and $q$ cannot be chosen arbitrarily. For instance, in the case of the 2-D oscillator, equation (7) can be solved in terms of $f$ :

$$
f=\frac{1}{4} V_{2} g^{2}-\frac{\left(V_{1} x_{2}+q(x)\right)}{V_{2}}
$$

From equation (18), it is clear that for continuous $g$, and $V \in \mathcal{C}^{1}$, we need:

$$
f(0)=-\lim _{x \rightarrow 0} \frac{\left(V_{1} x_{2}+q(x)\right)}{V_{2}}=0
$$

Furthermore, for $f$ to be continuous, we would impose:

$$
\frac{\left(V_{1} x_{2}+q(x)\right)}{V_{2}}<\infty, \quad \forall x
$$

Hence, for the 2-D oscillator, it only makes sense to consider $V$ and $q$ that satisfy the above two conditions, (19) and (20). Only then may we begin to consider the problem of using the converse approach to construct systems for which a certain design methodology happens to be optimal.

For instance, if we were considering JL for the 2-D oscillator (6), first we would need to consider its linearization at the origin:

$$
\begin{gathered}
\dot{x}=A x+B u \\
A=\left[\begin{array}{c}
-a_{1}- \\
-a_{2}-
\end{array}\right]=\left[\begin{array}{cc}
0 & 1 \\
\frac{\partial f(0)}{\partial x_{1}} & \frac{\partial f(0)}{\partial x_{2}}
\end{array}\right], B=\left[\begin{array}{c}
0 \\
g(0)
\end{array}\right] .
\end{gathered}
$$

Let

$$
P=\left[\begin{array}{l}
-p_{1}- \\
-p_{2}-
\end{array}\right]
$$

be the solution of the Ricatti equation:

$$
A^{T} P+P A-P B B^{T} P+Q=0
$$

where $q=x^{T} Q x+O\left(x^{3}\right)$. $V$ must be of the form,

$$
V=x^{T} P x+O\left(x^{3}\right) .
$$

since locally the system looks like its linearization, and hence, $V$ must locally look like the storage function for the linearized system (i.e. $x^{T} P x$ ).

To determine how to generate systems for which Jacobian linearization is optimal, we first design a locally 
optimal (i.e. for the nonlinear system linearized at the origin) controller using Jacobian linearization:

$$
u_{J L}=-B^{T} P x=-g(0) p_{2} x
$$

For this controller to be globally optimal, it must satisfy $u_{j l}=u^{*}$ or

$$
-g(0) p_{2} x=-\frac{1}{2} g V_{2}
$$

which gives $g=\frac{2 g(0) p_{2} x}{V_{2}}$ and from (18) yields:

$$
f=\frac{\left(g(0) p_{2} x\right)^{2}}{V_{2}}-\frac{V_{1} x_{2}}{V_{2}}-\frac{q(x)}{V_{2}}
$$

So, given admissible $V$ and $q$, as well as choosing $g(0)$, we use the quadratic portion of $V$ to determine $P$ and finally the above equations to determine $f$ and $g$ for which Jacobian Linearization will be optimal.

Example 2 was created in this way by choosing:

$$
V=2 x_{1}^{2}+\cos ^{2}\left(x_{1}\right)-2\left(1+x_{2}\right) e^{-x_{2}}+1, \quad q=x_{2}^{2}
$$

and $g(0)=1$.

In a similar manner, equations may be derived for other techniques. We briefly summarize a couple in the following table:

Table 2

\begin{tabular}{|c|}
\hline Jacobian Linearization \\
\hline$u^{*}=-g(0) p_{2} x$ \\
$g=\frac{2 g(0) p_{2} x}{V_{2}}$ \\
$f=\frac{\left(g(0) p_{2} x\right)^{2}}{V_{2}}-\frac{V_{1} x_{2}+q(x)}{V_{2}}$ \\
\hline Pseudo Jacobian Linearization \\
\hline$u^{*}=-g(x) p_{2} x$ \\
$g=$ arbitrary \\
$f=\frac{1}{2} p_{2} x g^{2}-\frac{V_{1} x_{2}+q(x)}{2 p_{2} x}$ \\
\hline Feedback Linearization \\
$u^{*}=-(1 / g)(f+k x)$ \\
$g^{2}=\frac{4\left(\left[g(0)^{2} p_{2}-a_{2}\right] x\right)}{V_{2}}-\frac{4}{V_{2}^{2}}\left(V_{1} x_{2}+q(x)\right)$ \\
$f=\left[g(0)^{2} p_{2}-a_{2}\right] x-\frac{2}{V_{2}}\left(V_{1} x_{2}+q(x)\right)$ \\
\hline
\end{tabular}

Note that each of the above equations places even further restrictions on admissible $V$ and $q$. For instance, in $\mathrm{JL}$, we would like that $V_{2}$ divides $p_{2} x$. In pseudo $\mathrm{JL}\left(u^{*}=-g(x) p_{2} x\right)$, any admissible $V$ of the form $V=x^{T} P x+O\left(x_{1}^{3}\right)$ will work (i.e. $V_{2}=2 p_{2} x$ ). Similar to the 1-D case, we find that FL seems to have more restrictive conditions then the others. In addition to choosing $V$ and $q$ so that the resulting $f$ and $g$ are continuous, we need $g>0$ for all $x$ so that the system will be feedback linearizable. This can be a tricky process. Finally, it is interesting to note that for $V$ and $q$ quadratic, $f$ and $g$ can only come from a linear system for JL or FL to be optimal.

\section{References}

[1] Basar, T. and P.Bernhard, $\mathcal{H}_{\infty}$-Optimal Control and Related Mini-Max Problems, Berlin: Birkhauser, 1991.

[2] Cloutier, J.R., C. N. D'Souza, and C. P. Mracek, "Nonlinear Regulation and Nonlinear $H_{\infty}$ Control via the State-Dependent Riccati Equation Technique," Preprint.

[3] Crandall, M.G. and P.L. Lions, "Two Approximations of Solutions of Hamilton-Jacobi Equations," Mathematics of Computation, Vol.43(167), pp.1-19.

[4] Huang, J. and C.-F. Lin, "A Numerical Approach to Computing Nonlinear $H_{\infty}$ Control Laws," J. Guidance Contr. Dynamics, to appear.

[5] Huang, Y. and W.-M. Lu, this session.

[6] James, M.R. and S. Yuliar, "Numerical Approximation of the $H_{\infty}$ Norm for Nonlinear Systems," $A u$ tomatica, 1995.

[7] Lu, W.M. and J.C.Doyle, " $\mathcal{H}_{\infty}$-Control of Nonlinear Systems: A Class of Controllers", Caltech CDS Tech. Memo., No. CIT-CDS-93-008, also Proc. 1993 IEEE CDC, San Antonio, TX, 1993.

[8] Lu, W.M. and J.C.Doyle, " $\mathcal{H}_{\infty}$-Control of Nonlinear Systems: A Convex Characterization", IEEE Trans. $A C$, Vol.40(9), pp.1668-1675.

[9] Van der Schaft, A.J., "Nonlinear State Space $\mathcal{H}_{\infty}$ Control Theory", Perspectives in Controlleds. H.L.Trentelman and J.C.Willems), Birkhauser, 1993.

[10] Willems, J.C., "Dissipative Dynamical Systems", Arch. Rat. Mech. Anal., vol.45, pp.321-393, 1972.

A life spent in making mistakes is not only more honorable but more useful than doing nothing.

George Bernard Shaw 